\title{
Apoptosis, Angiogenesis and Cancer Therapies
}

\author{
Manjul Tiwari*
}

\begin{abstract}
Angiogenesis, the growth of new blood vessels from the existing vasculature, and is maintained in adult tissues by the balanced presence of both angiogenic inducers and inhibitors in the tissue milieu. When inducers predominate, vascular endothelial cells (VECs) become activated and in this activated VECs, distinct cell signaling pathways are initiated providing the specificity of anti-angiogenic therapies to the tumor vasculature. VEC apoptosis has been well documented in regressing vessels, and it has been shown that, in addition to activating the VECs, some inducers such as vascular endothelial growth factor also up-regulate Fas expression, thus sensitizing the cell to apoptotic stimuli. Endogenous angiogenesis inhibitors, such as thrombospondin-1(TSP-1) and pigment epithelium-derived factor (PEDF), stimulate signaling cascades within the VECs and also induce the expression of Fas ligand in activated VECs. Therefore, when inhibitors predominate, the apoptotic cascade is initiated ,thus anti-angiogenic therapies can target the inducer supply or directly target the VECs. Although clinical studies suggest that anti-angiogenic therapies may prove to be most effective when used in combination with traditional therapies [1].
\end{abstract}

\section{Introduction}

\section{Angiogenesis and Tumor Growth}

Angiogenesis, is a normal and necessary process during growth and development. It also occurs in the adult during wound healing and the reproductive cycles. However, in most normal adult issues, the vasculature is maintained in a quiescent state by the balanced presence of both pro-angiogenic and anti-angiogenic molecules in the tissue milieu [1]. Vascular endothelial cells (VECs) have a very low turn over time, estimated any where from 1000 days $[1,2,3]$ to 7 years $[1,4]$. Judah Folkman first proposed in 1971 that tumor growth is dependent on the induction of angiogenesis $[1,5]$.

It is demonstrated that this is due to a balance between tumor cell proliferation and apoptotic rates which applies to micrometastases, which remain dormant until a pro-angiogenic environment arises $[1,6,7,8]$. Tumors shift the balance to favor angiogenic induction by increasing expression of angiogenic inducers, decreasing expression of angiogenic inhibitors, or a combination of both $[1,9]$ which result from the same genetic and epigenetic changes that drive tumor progression, such as oncogene expression, loss of tumor suppressor gene expression, and tissue hypoxia [1,9-12]. Through tumor cell stimulation angiogenic balance can indirectly altered to produce angiogenic factors and/or to decrease inhibitor production $[1,13]$.

\footnotetext{
*Correspondence: manjultiw@gmail.com

Department of Oral Pathology and Microbiology, School of Dental Sciences, Sharda University, Greater Noida, India.
}

Neovascularization can also further stimulate tumor growth by providing the tumor with paracrine growth factors secreted by the VECs themselves, such as basic fibroblast growth factor (bFGF), interleukin-6, insulin-like growth factor, and platelet-derived growth factor, and also factors released by macrophages or other blood cells delivered to the tumor through the vasculature [1,13-16]. Furthermore, these vessels provide an avenue for tumor cells to metastasize to distant locations. Thus, the transition to a pro-angiogenic phenotype provides significant advantages for tumor growth and metastases [1].

\section{Angiogenesis ,Tumor Vasculature and Cancer Therapies [1] When the balance of angiogenic mediators shifts to favor angiogenic induction, VECs become activated, resulting in changes in gene and protein expression patterns. Proteases released by the activated VECs degrade the basement membrane, allowing migration toward the angiogenic inducer source. VEC proliferation subsequently ensues, followed by lumen formation $[1,17]$. In addition, coculture experiments of tumor cells with one type of VEC, human umbilical vein endothelial cells, have demonstrated coordinated increases in both expression of specific ligands and their receptors in the endothelial cells in response to the tumor cell secretions, creating autocrine stimulation of the VECs $[1,18]$, thus further promoting angiogenesis[1].}

(c) 2012 Tiwari; licensee Herbert Publications Ltd. This is an open access article distributed under the terms of Creative Commons Attribution License (http://creativecommons.org/licensesby/2.0), This permits unrestricted use, distribution, and reproduction in any medium, provided the original work is properly cited 
The tumor vessels formed are markedly different than the vessels in normal tissues and organs. They are associated with vascular leakage and the presence of inflammatory cells $[1,17,19]$. In addition, they often do not have associated pericytes and have reduced basement membrane formation, likely contributing to their leakiness $[1,17]$ which provides the specificity of anti-angiogenic therapies for the activated VECs associated with the tumor vessels [1].

It is important to note that apart from cancer treatment by supplementing surgergy, chemotherapy or radiation therapy angiogenic therapies (both pro and antiangiogenic agents) have potential therapeutic benefit in other diseases as well. In principal, anti-angiogenic cancer therapies offer several advantages over the traditional cytotoxic chemotherapies and radiation therapies. First, they are not mutagenic, so secondary tumors are unlikely $[1,9,20-22]$. Second, they target the tumor vasculature specifically; therefore, they do not cause the side effects associated with traditional chemotherapies, such as nausea, hair loss, and bone marrow suppression [1,19,22]. Third, they can act synergistically with current chemotherapies, radiation and gene therapies [1,23-27].

Fourth, they can be easily delivered through the circulation to their target cells $[1,9,22]$. Last, as they do not target the tumor cell, but instead the genetically stable VECs, the tumor cells are unlikely to develop resistance to therapy $[1,28,29]$ However, in this last advantage also lies a disadvantage. As the VECs, and not the tumor cells, are the target, these therapies do not kill the tumor cells directly and are therefore not curative [1].

With these therapies, theoretically, the tumor would be reduced to avascular size limits. Thus, to overcome this issue, two approaches have been taken. One approach is to view anti-angiogenic therapies as chronic, long-term therapies. Another approach is to combine anti-angiogenic therapies with cytotoxic therapies that do directly target the tumor cell[1].

Most data to date suggest that anti-angiogenic therapies are more effective when administered on a more frequent, or metronomic, low dose schedule $[1,10]$. Folkman $[1,2,17,19,22]$ prefers the term "anti-angiogenic schedule" to describe this type of dosing to emphasize that the VECs are the target of the treatment rather than the tumor cells, as metronomic implies only a regular interval of dose delivery; however, the term metronomic is most often used[1]. The maximum tolerated dose (MTD) schedule appears to allow for recovery of the tumor vessels between treatments and thus does not effectively suppress vessel growth. The continuous presence of the anti-angiogenic agent prevents vascular re-growth, therefore, suppressing tumor growth more efficiently $[1,31]$. With several advantages anti-angiogenic agents have fewer side effects in general, especially when used on lower, metronomic dosing schedules, it is feasible that antiangiogenic agents could be developed as long-term, chronic therapies rather than acute therapies, such as done for the treatment of high blood pressure or high cholesterol [1].

In support of this hypothesis, some chemotherapeutic drugs have been found to have anti-angiogenic activity, when used on metronomic dosing schedules. Kerbel and colleagues called such chemotherapeutic drugs "accidental" anti-angiogenic drugs for example, vinblastine and cyclophosphamide (CPP) [1,32-38]. Using chemotherapeutic agents on metronomic dosing schedules offers several advantages over traditional dosing schedules in that side effects are greatly reduced and tumor resistance is less likely[1].

To date, many antiangiogenic agents when used alone, even on metronomic scheduling, have not shown significant efficacy in the majority of patients or take an extended period of time to achieve significant tumor regression, even up to 1 year or more [1,28,31]. Teicher and colleagues [1] were the first to pioneer to combined treatment modality (metronomic chemotherapies combined with second agent) and produced several convincing studies demonstrating synergy between various anti-angiogenic therapies and various cytotoxic therapies in mouse models. One caveat, however, is that when anti-angiogenic agents were used with chemotherapies on a MTD (Maximum Tolerated Dose) schedule, the combination produced some serious side effects $[1,39]$. Therefore, combining anti-angiogenic therapies with chemotherapies that have demonstrated anti-angiogenic function and efficacy on a low dose, metronomic schedule, rather than a MTD schedule, may prove to be the safest and most effective approach $[1,33,35]$. Based on the animal studies, it appears that combining anti-angiogenic agents with traditional cytotoxic therapies may be the most promising treatment avenue, and this is being actively pursued in clinical trials [1].

\section{Endothelial Cell Apoptosis in Cancer Therapy and Activated VECs[1]}

To inhibit angiogenesis, an anti-angiogenic agent could target the vecs at any of the steps necessary for neovascularization, such as extracellular matrix degradation, migration, proliferation, or tube formation. For example, platelet factor-4 inhibits endothelial cell proliferation whereas tissue inhibitor of matrix metalloproteinases- 2 inhibits extracellular matrix degradation, tube formation, and VEC proliferation [1,21,27].

Therefore, it is reasonable that an angiogenesis inhibitor functions by actively promoting apoptosis of VECs. In vitro studies have shown that many endogenous angiogenesis inhibitors do indeed induce apoptosis, including TSP-1, pigment epithelium-derived factor (PEDF), prolactin, angiostatin isoforms, endostatin, tumstatin, and canstatin, 2-methoxyestradiol, and doxorubicin [1,9,11,18,21,27,27].

Many studies have demonstrated VEC apoptosis in vivo using animal tumor models, and in the prostate, in particular, the vasculature has been intensely studied for its involvement in prostatic growth regulation. In castrated rats, which have an atrophied prostate, testosterone treatment increases VEGF expression and stimulates angiogenesis prior to prostatic re-growth, and androgen ablation therapy also appears to target the vasculature. In both the normal prostate and prostate tumor models, androgen ablation therapy increases VEC apoptosis, reduces blood flow, and results in vascular regression, and the VEC apoptosis precedes the epithelial cell apoptosis and prostate tissue regression . In both rodent 
and human prostate tissues, in response to androgen ablation therapy in the normal and tumor epithelial cells as well as stromal cells, VEGF expression is decreased and the expression of the angiogenesis inhibitors PEDF. The changes in expression of VEGF, TSP-1, and PEDF, in response to androgen withdrawal, likely shift the angiogenic mediator balance thus triggering VEC apoptosis and vascular regression. These studies support a critical role for angiogenic regulation in normal and malignant prostate growth and emphasize the role of VEC apoptosis in growth regulation $[1,11,27]$.

It is well established that endothelial cell survival depends on the balance of both stimulatory and inhibitory signals in the extracellular milieu. When a predominance of inducers is present, the VECs[1,32] become activated, with subsequent changes in gene and protein expression profiles. There are many angiogenic inducers now known however, of these, VEGF seems to be up-regulated in a wide range of tumor types and thus has been the most intensely studied in tumor biology $[1,21,29]$. Therefore, here it is focus on the pathways stimulated by VEGF in the VECs. It is important to note, however, that pathways stimulated by other growth factors and molecules overlap with those stimulated by VEGF. VEGF is a member of the VEGF family of related proteins and is denoted as VEGF-A or simply VEGF. It is produced as several isoforms resulting from alternative splicing, with the most abundant ones being VEGF121, VEGF165 (most prevalent isoform and is overexpressed in many tumor types), VEGF189, and VEGF206 [1,29].

There are several VEGF receptors on VECs, including receptors 1 and 2 (VEGFR-1 and VEGFR-2) and neuropilin-1. VEGFR-1 (also called flt-1) primarily plays a regulatory role in VECs, as a "decoy" receptor, rather than a signal transduction role [1,8,29]. Neuropilin-1 in VECs $[1,7,9,27,32]$, intracellular signaling has not been demonstrated, but it may function to stabilize the VEGF/ VEGFR-2 complex. VEGFR-2 (also called flk-1 or Kinase insert domain receptor (KDR)) is expressed on the surface of most VECs and is primarily responsible for VEGF signal transduction $[1,8,30]$. VEGF binding to VEGFR-2 stimulates receptor dimerization and auto-phosphorylation of tyrosine residues in the cytoplasmic tail, which then triggers the phosphorylation of $\mathrm{SH} 2$-domain containing proteins. This initiates multiple signaling cascades within the VECs ultimately supporting survival, mitogenesis, migration, and tube formation $[1,8,36,38]$.

VEGF also directly inhibits apoptosis through induction of expression of anti-apoptotic proteins such as bcl-2, survivin and $A 1$, and repression of expression of pro-apoptotic proteins such as Bad, Bax, and caspase-9 [1]. In addition, through the $\mathrm{PI} 3 \mathrm{~K} / \mathrm{Akt}$ pathway, VEGF also stimulates expression of the antiapoptotic Flice-like inhibitor protein (FLIP) [1,29]. FLIP inhibits the activation of procaspase-8, and cells expressing elevated levels of FLIP are resistant to Fas-mediated apoptosis $[1,38]$.

Therefore, the quiescent VECs are resistant to the effects of circulating angiogenesis inhibitors because they do not express Fas at high levels, whereas the activated VECs are sensitized to respond to apoptotic stimuli. Therefore, if the balance shifts such the angiogenesis inhibitors predominate within the tumor microenvironment, apoptosis will be initiated in the activated VECs. The differences between quiescent and activated VECs provide the specificity that anti-angiogenic therapies have for activated VECs associated with the tumor vasculature[1,7-9,27,29,30,32,36,38].

\section{p53-Dependent Apoptosis Induced by DNA Damage}

p53 is a transcription factor for a set of pro-apoptotic proteins from the Bcl-2 family (Bax, Bid, Noxa and Puma) that promote mitochondrial permeabilization, and thereby the release of cytochrome c. p53 also induces ASC (apoptosis-associated speck-like protein) that relocalizes Bax to mitochondria [1,15].

Several other mitochondria-targeting proteins are induced by p53. Some of these, such as ferrodoxin reductase, are involved in the production of reactive oxygen species (ROS), while others permeabilize mitochondria either directly or indirectly by less understood mechanisms. These proteins include p53AIP (p53-regulated apoptosis-inducing protein-1), mitochondrial chloride intracellular channel-4, PIDD (p53-induced protein with a death domain; implicated in caspase-2 activation;) and histone H1.2. p53 also transactivates components of the transmembrane "extrinsic" pathway (e.g., Fas/CD95, DR5 and PERP), proteins located to the endoplasmic reticulum, caspase- 6 and the procaspase-9 adapter Apaf-1. p53 also represses the transcription of the anti-apoptotic proteins $\mathrm{Bcl}-2$ and survivin (an IAP that prevents caspase activity and regulates cell cycle) $[1,6,9,25,27]$.

p53 also induces apoptosis in a transcription-independent manner by directly targeting mitochondria. After DNA damage, a fraction of p53 is exported from the nucleus and binds to the outer mitochondrial membrane. Mitochondrial p53 binds to $\mathrm{BCl}-2$ and $\mathrm{BCl}-\mathrm{xL}$ and neutralizes their inhibitory effect on Bak (and Bax), resulting in Bak oligomerization and subsequent mitochondrial permeabilization $[1,18]$.

$\mathrm{BCl}-2$ and $\mathrm{BCl}-\mathrm{xL}$ interact with the DNA-binding region of $\mathrm{p} 53$, the same region that harbors the vast majority of "hot spots" mutations in human cancer cells. Thus, some p53 mutations that interfere with DNA binding also interfere with $\mathrm{p} 53$ binding to $\mathrm{BCl}-2$ and $\mathrm{BCl}-\mathrm{xL}$.

It has been proposed that $\mathrm{p} 53$ triggers a rapid first wave (within minutes) of transcription-independent apoptosis that precedes a second slower/delayed (within hours) transcriptiondependent wave. The importance of p53 mitochondrial translocation in the response to DNA damage is controversial. The direct mitochondrial effect of p53 is cell type dependent, as wild-type $\mathrm{p} 53$ in primary fibroblasts does not translocate to mitochondria whereas wild-type p53 in thymocytes does. The molecular basis of this difference is not known. In addition, p53 itself can bind to DNA strand breaks $[1,19]$ and might therefore be involved also in DNA damage detection/repair[1].

Histone $\mathrm{H} 1.2$ also relocalizes to mitochondria following DSBs. This process requires $\mathrm{p} 53$ but is independent of $\mathrm{p} 53$ transcriptionnal activity. $\mathrm{H} 1$ is the histone subunit that binds the chromatin linker region between the nucleosomes. Humans and mice have eight histone $\mathrm{H} 1$ genes. Among them, all of the somatic $\mathrm{H} 1 \mathrm{~s}(\mathrm{H} 1.1-\mathrm{H} 1.5)$ are ubiquitously expressed in all tissues throughout development. After DSBs, all nuclear histone $\mathrm{H} 1$ variants relocate partially to the cytoplasm. However, only H1.2 is able to trigger cytochrome $\mathrm{c}$ release from mitochondria. 
The mechanism for the nucleo-cytoplasmic redistribution of $\mathrm{H} 1.2$ is unclear as $\mathrm{H} 1.2$ does not undergo any obvious posttranslational modification, neither change in expression. DSBs themselves or the subsequent $\mathrm{p} 53$-dependent repair process may cause $\mathrm{H} 1.2$ release from chromatin to the nucleoplasm and subsequently in the cytoplasm. At the mitochondrial level, $\mathrm{H} 1.2$ induces conformational activation and oligomerization of Bak. In mitochondria isolated from Bak-deficient cells, $\mathrm{H} 1.2$ fails to induce cytochrome $\mathrm{c}$ release. The mechanism for $\mathrm{H} 1.2$ to cause the conformational change of Bak and the subsequent cytochrome $c$ release remains to be determined. H1.2 appears to respond specifically to DSB-induced apoptosis as down-regulation of $\mathrm{H} 1.2$ by antisense RNA or small interfering RNA (siRNA) reduces apoptosis induced by X-rays or the Top2 inhibitor etoposide, but not by tumor necrosis factor- or UV radiations. Also, thymocytes and cells in the small intestine from $\mathrm{H} 1.2$-deficient mice show strong resistance to $X$-ray-induced apoptosis $[1,6,9,10,15,19,28,30]$.

Procaspase-2 might also link DNA damage and mitochondria [1,22]. The "PIDDosome," a molecular complex containing PIDD, whose expression is induced by p53, and RAIDD/CRAIDD, an adaptor protein with a death domain, activate procaspase- 2 in the nucleus $[1,23]$. Increased PIDD expression results in spontaneous activation of procaspase-2 and sensitization to apoptosis by genotoxic stimuli $[1,23]$.

In the nucleus, juxtaposition of procaspase-2 molecules (dimerization) results in autocleavage/activation [1,24], a similar mechanism of activation to the initiator procaspase- 8 and procaspase-9. Release of mature caspase- 2 can stimulate directly mitochondrial release of cytochrome c. This process requires the processing of procaspase- 2 but not its enzymatic activity and is also independent of Bax, Bak and Bcl-2 [1,25].

p53 can activate multiple pathways besides apoptosis, including cell cycle arrest/checkpoint, DNA repair, senescence and angiogenesis. Both cell cycle arrest and apoptosis prevent replication of damaged DNA and represent a coherent response for $\mathrm{p} 53$ as "the guardian of the genome." However, the p53-mediated cell cycle arrest response might in some cases antagonize p53-mediated apoptotic response. For instance, activation of p21CIP1, which was among the first isolated p53-dependent genes, induces both cell cycle arrest in response to low doses of camptothecin and blocks DNA damage-induced apoptosis $[1,26,27]$. p53 may selectively induce apoptosis in cells with elevated E2F1 activity, such as pRb-deficient cells. p53 binds to the cyclin A box of E2F1, and this complex induces apoptosis when cyclin $A$ is low $[1,28]$. E2F1, like p53, is negatively regulated by $\mathrm{Mdm} 2$, and both $\mathrm{E} 2 \mathrm{~F} 1$ and $\mathrm{p} 53$ are up-regulated in response to DNA damage $[1,29]$. p53 may also specifically promote apoptosis when it is transcribed as a N-terminal truncated variant, designated p53/47 [1].

In contrast to p53, p53/47 lacks the Mdm2-binding domain. Thus $M d m 2$ expression increases the ratio p53/47 to $p 53$. p53/47 has a different gene expression profile: up-regulation of Bax and down-regulation of p21 $[1,30]$. It is therefore possible that specific modifications of p53 might selectively activate apoptotic or cell cycle arrest genes. Depending on the cellular context, p53 might selectively activate one set of genes or the other. The pleiotropic regulation of p53 could allow fine tuned adjustments of p53 levels and p53 phosphorylation (depending on the intensity of DNA damage), which could account for the selectivity of p53 for transactivating cell cycle arrest and/or apoptotic genes [1].

\section{p53-Independent Apoptosis Induced by DNA Damage[1]}

In spite of the apparent pivotal role of p53 in apoptosis [1,32,33], p53-null cells such as HL60 and U937 human leukemic cells undergo apoptosis readily in response to DNAdamaging agents [1,34-36]. In these cells, the p53-related protein $\mathrm{p} 73$ does not compensate the lack of $\mathrm{p} 53$ as apoptosis is also transcription independent. Moreover, more than $50 \%$ of human tumors contain mutated and defective p53. Although such tumors might be defective in their apoptotic response in vivo, experiments performed in cell cultures demonstrate that these tumors can undergo apoptosis in response to Top1 poisons [1,37]. p53-independent apoptosis involves the receptor TR3 and the checkpoint kinase Rad9 that target directly mitochondria in response to DNA damage and also the DNA repair protein Ku70. Other p53-independent pathways include Chk2, E2F1, PML and c-Abl [1].

The orphan receptor TR3 (also called Nur77 or nerve growth factor-induced protein B) is a transcription factor of the steroid/thyroid receptor superfamily. TR3 is involved in promoting cell proliferation $[1,38]$. It is also a critical inducer of apoptosis.

TR3 gene is rapidly induced by different apoptosisinducing agents, and overexpression of a dominantnegative TR3 protein [1] or inhibition of TR3 expression by antisense TR3 mRNA $[1,40]$ inhibits apoptosis. By contrast, constitutive expression of TR3 induces apoptosis. Although the mitogenic effect of TR3 occurs in the nucleus through target gene regulation, the pro-apoptotic effect of TR3 occurs in the cytoplasm independently of its transactivating activity. In response to apoptotic stimuli (including DSBs), TR3 translocates from the nucleus to mitochondria where it induces the mitochondrial release of cytochrome c. Despite lacking classical mitochondria-targeting sequences, TR3 relocates to mitochondria by binding to the $\mathrm{BCl}-2 \mathrm{~N}$-terminal loop region. The TR3- $\mathrm{BCl}-2$ interaction induces a $\mathrm{BCl}-2$ conformational change that exposes its $\mathrm{BH} 3$ domain [1]. TR3 could therefore act on mitochondria by converting $\mathrm{Bcl}-2$ from an anti-apoptotic to a pro-apoptotic member of the $\mathrm{Bcl}-2$ family [1].

Another molecule that directly targets mitochondria is the cell cycle checkpoint Rad9. Rad9 is loaded as a complex with Hus1 and Rad1 (known as 9-1-1 complex) onto damaged chromatin by a clamp loader consisting of Rad17 and replication factor $C$. The 9-1-1 complex promotes the phosphorylation/activation of Chk1 by ATR, which in turn induces cell cycle arrest. Following DNA damage, Rad9 can also migrate from the nucleus to mitochondria. The $\mathrm{BH} 3$-like domain of Rad9 interacts with $\mathrm{BCl}-2$ and $\mathrm{BCl}-\mathrm{xL}$, which induces 
apoptosis . Dual phosphorylation of Rad9 by c- Abl [1,4,6] (which is itself activated by ATM) and PKC may be required for Rad9 to bind $\mathrm{BCl}-2$ and $\mathrm{BCl}-\mathrm{xL}$.

Ku70 is another molecule involved in both DNA repair and apoptosis. In addition to its nuclear localization, Ku70 is present in the cytoplasm where it binds to Bax, preventing Bax mitochondrial localization and pro-apoptotic activity $[1,4,15]$.

Bax-mediated apoptosis is suppressed by overexpression of Ku70. By contrast, Bax-mediated apoptosis is enhanced by down-regulation of Ku70. Upon apoptotic stimuli, Bax is released from Ku70. Acetylation of Ku70 by p300/CBP and PCAF/ GCN5 may induce this dissociation. As a result, the $\mathrm{N}$-terminus region of Bax is exposed, allowing Bax to form a large complex, called the "baxosome," containing Bax itself, $\mathrm{BH} 3$-only proteins, cardiolipin, and probably other unidentified proteins [1].

In the baxosome, Bax undergoes conformational changes, allowing its insertion into the outer membrane mitochondrial, where it promotes the release of cytochrome $\mathrm{c}$ and other proapoptotic molecules. Because Ku70 accumulates following DSBs, it is possible that Ku70 would prevent Bax from inducing a premature apoptosis (if the cell can still repair). However, beyond a certain threshold of DNA damage, Ku70 would release Bax to induce apoptosis.

The relative importance of these pathways in p53-deficient cells is not known. It will be interesting to use genetically altered cells and/or selective pharmacological inhibitors to determine the relative contribution of each of these pathways.

\section{Role of Extrinsic Pathway in Chemotherapy and Radiosensitivity[1]}

Chemotherapy and radiation, when used successfully, act to inhibit tumor growth. lonizing radiation and DNA-damaging chemotherapeutics can elicit an apoptotic response that is principally mediated through activation of the p53 tumor suppressor protein. p53 is the most commonly mutated protein found in human cancers and is a potent transcriptional activator of genes that play principal roles in cell-cycle arrest and apoptosis [1-3]. Recent evidence suggests that p53 also influences apoptosis by directly interacting with members of the $\mathrm{Bcl}-2$ family. Members of the $\mathrm{Bcl}-2$ family that p53 can activate transcriptionally include Bax, Puma, Noxa, Bnip3L, Bak, and Bid. p53 also directly contributes to activation of the extrinsic pathway. Death receptors for both TRAIL and FasL have been identified as p53 target genes[1].

KILLER/DR5 was originally discovered as a DNA-damageinducible p53 target gene and is transcriptionally activated by $p 53$. Certain tissues, including the spleen, small intestine, and thymus, show large increases in DR5 expression following ionizing radiation that is dependent on transcriptionally active p53 [1,8,15,27].

DR4 may also be regulated by $\mathrm{p} 53$ in a limited number of cell lines $[1,28]$. Several studies have found a p53-dependent increase in Fas or FasL, which contributes tomediating the apoptotic response after conventional chemotherapy, but Fas is not essential for mediating p53's effects. Lymphocytes from Ipr mice, or those expressing DN-FADD, are equally sensitive to chemotherapy and ionizing radiation; p53 deficiency or constitutive expression of $\mathrm{Bcl}-2$ markedly increased the resistance of lymphocytes to gamma radiation or anticancer drugs, but lymphocytes were still sensitive to killing by FasL. Furthermore, apoptosis induced by chemotherapeutic drugs is not altered in embryonic fibroblasts from FADD and caspase 8 knockout mice, indicating only a partial role for the death receptor pathway in response to chemotherapeutic agents. Nevertheless, partial resistance of DR5-null tissues to ionizing radiation implicates the extrinsic pathway in DNA-damageinduced apoptosis[1].

\section{Involvement of Cell Death during Tumor Development and Treatment[1]}

From this brief overview, it is already clear that various mechanisms of cell death can contribute to cancer development and treatment response in largely varying degrees.

The loss or down regulation of cell death pathways clearly occurs during cancer development, not only in the case of apoptosis but in other mechanisms of cell death. In the case of cancer development, it is easy to see how even subtle changes in cell death can contribute to tumor growth and development. A carefully regulated balance between cell proliferation and cell death is absolutely required for maintenance of tissue homeostasis. This equilibrium needs to be only modestly affected to allow proliferation to exceed cell death and consequently to the development of a tumor. Selection against apoptosis may be especially important, as many oncogenes not only promote proliferation but also sensitize the cell to death by apoptosis $[1,8]$. Consequently for the cell to realize the true potential of an oncogene, it must also counter-balance this increased apoptotic sensitivity.

However, it is less clear whether the changes in sensitivity to cell death that occur during carcinogenesis will also significantly alter the response of these same tumors to treatment. There are certainly compelling experiments that have clearly demonstrated that apoptosis can influence tumor response.

Tumors arising because of the presence of myc activation in the B-cell lineages are highly influenced by secondary pathways that affect apoptosis. For example, when these mice are crossed with $\mathrm{p} 53+$ /- heterozygous mice, all the lymphomas that develop demonstrate loss of heterozygosity for p53 [1,34]. Furthermore, loss of apoptosis through knockout of p53, overexpression of BCL-2, or loss of INK4A/ARF dramatically accelerates tumor onset and tumor growth. This model clearly demonstrates that apoptosis acts as a barrier to cancer development. This model has also provided evidence that apoptosis is important for cancer therapy. When mice-harboring lymphomas were treated with chemotherapy agents, the tumors with loss of $\mathrm{p} 53$, INK4A/ARF, or overexpression of BCL2 also responded much more poorly than those expressing only myc. These data clearly demonstrate that in this model, the propensity to undergo apoptosis influences tumor response [1].

Despite this compelling data, it is not necessarily extrapolate this result to human tumors, especially those of non-lymphoid origin. It is described above displays an extreme sensitivity 
to apoptosis because of two distinct factors. First, the cells of lymphoid origin generally show a much higher propensity to die by apoptosis-in other words, the balance between pro-and anti-apoptotic proteins is already tipped in favor of apoptosis in these cells. Second, overexpression of myc also results in an increased sensitivity toward apoptosis. Consequently, the control tumors in this case are extremely sensitive to apoptosis. This is perhaps best exemplified by the fact that a single dose of cyclophosphamide is sufficient to cause a long-term remission in the majority of mice. In solid human tumors, which develop primarily from epithelial cells, the situation may be substantially different. Indeed, in a comprehensive review of the literature, Brown and Wilson concluded that "there is little or no support that apoptosis, and the genes govern it, determine the response to therapy" $[1,9]$. In contrast to the results with the lymphoma model, they found that the propensity to undergo apoptosis in human tumors of epithelial origin played no role in predicting treatment sensitivity.

Despite the aforementioned review of clinical data, the concept that selection of cells with resistance to apoptosis during carcinogenesis results in a co-selection of cells that will be resistant to treatment has become a persuasive one in the research community. It is important to evaluate all potential forms of cell death that may contribute to treatment response. Furthermore, for each type of cell death, it is critical to consider both the kinetics of cell death and its dose-response relationship with the treatment [1].

\section{Laboratory Evaluation of Treatment Responses and their Interpretations}

One of the principle reasons for the widely held view that apoptosis plays an important role in treatment sensitivity arises from the use in vitro and in vivo assays that are biased or inappropriate for assessing overall treatment sensitivity. Outlined below is a brief description of some of these assays and their strengths and limitations with respect to evaluation of treatment response.

\section{In Vitro}

A number of in vitro assays are used to assess treatment sensitivity, including those based primarily on the cell growth. The MTT assay, which measures the activity of a mitochondrial enzyme, or assays based on cellular protein or DNA content are often considered measures of viability and surrogates for treatment sensitivity. However, these assays are chiefly based on measurements of cell number. These assays are normally executed several days after exposure of cells to a damaging agent and as such are influenced not only by cell death but also by transient changes in the rate of cell growth. As many of the same genes that influence cell death also influence cell proliferation, especially in the case of apoptosis, it is difficult or impossible to interpret overall treatment sensitivity from these types of assays. A good example is data from the $\mathrm{NCl} 60$ cell line database suggesting that $\mathrm{p} 53$ is a strong predictor of treatment sensitivity. In this study, treatment sensitivity was evaluated by a short-term (2-day) growth assay. As p53 elicits temporary cellcycle blocks in response to DNA damage, it is not surprising that this assay would produce such a result. Regardless of how it is performed, any assay that is performed at such short times after treatment will by definition ignore any forms of cell death that occur after longer times. Indeed, critical analysis of the role of p53 in response to radiation and other treatment stresses has shown that it is not a reliable predictor of response [1].

As an alternative, several in vitro assays are based on the detection of specific modes of cell death including apoptosis. The least effective assays do not evaluate treatment at the cellular level but instead measure an average or population response. These assays include those that measure changes in the average level of caspase activity or the amount of DNA fragmentation. These assays are useful to demonstrate that apoptosis is occurring but are relatively non-quantitative. Somewhat better are cell-based assays for apoptosis that evaluate the known features of apoptosis such as DNA condensation or exposure of phosphatidyl serine at the cell surface. Other modes of cell death can similarly be identified using assays based on the known morphological and/or biochemical features of that particular form of cell death. For example, senescence is often detected by an increase in cellular-galactosidase activity [1].

There are two fundamental limitations of all these deathbased assays that limit their usefulness as measures of treatment sensitivity. The first is that they give a picture of the response only at the point in time-the time at which the cell population is evaluated. Given the fact that cells in a population can die by several different processes that each operate with different kinetics that may further vary in different cell types, one can never be sure that the cells that remain viable at the time of assessment will not subsequently die by some form of cell death. This can be partially solved by making continuous measurements as a function of time. However, this introduces further complication as surviving cells begin to proliferate and dilute the dead cells [1].

Furthermore, as the end stages of apoptosis can result in complete cell destruction, the apoptotic cells are also eliminated from assessment as time progresses. This is particularly a problem when attempting to assess cell death by these assays in vivo. The second major problem is that it is difficult or perhaps even impossible to simultaneously assess all possible modes of cell death. Consequently, these death-based assays can never give a full picture of treatment response [1].

The solution to the problem of identifying all forms of cell death was solved in 1956 by Puck and Markus, who developed an assay based on the ability of a single cell to grow into a colony. This "clonogenic assay" has formed the basis of in vitro cellular response studies in tumors and also some normal tissues. The clonogenic assay tests for the ability of a cell to recover from treatment in such a way that it can proliferate again and form a clone of substantial size (normally evaluated 10-20 days after treatment). It thus can measure the ability of a cell to survive from all possible (known and unknown) forms of 
cell death. This assay is analogous to the well-accepted and wellproven assays of treatment sensitivity in other organisms such as yeast and bacteria [1].

Clonogenic survival shows a typical log-linear relationship with treatment dose implying that the probability of cell kill increases in a roughly linear way with dose. In other words, each incremental increase in dose kills the same percentage of remaining cells. This relationship is well supported by in vivo dose responses of tumors containing large numbers of cells, which correspondingly require a very low percentage of cell survival for cure. The relationship between treatment dose and clonogenic survival established in vitro has successfully predicted the doses required to cure transplanted tumors in vivo even when rates of apoptosis do not correlate. For example, given the fact that a $1 \mathrm{~cm} 3$ tumor contains more than 109 cells, treatment requires killing more than $99.9999999 \%$ of the cells to have a chance at being effective. The log-linear relationship of cell survival and treatment dose allows one to predict the curative doses required to reach these levels [1].

This numerical example also exemplifies the importance of comparing dose-response relationships for the various death processes when evaluating their relative importance. For example, if a particular treatment dose results in $0.1 \%$ survival (99.9\% death) as assessed by a clonogenic assay, but causes only $35 \%$ apoptosis, one can conclude that apoptosis is not an important contributor to cell death. The fact that $99.9 \%$ of cells are killed implies that cell death is equally effective in the $65 \%$ of cells that do not die by apoptosis. One can easily imagine in this case that substantial increases or decreases in apoptosis induction would not affect clonogenic survival [1].

The literature is full of examples in which modest levels of cell death are assessed using treatment doses that will inactivate several logs of cell kill if assessed by the clonogenic assay. Obviously, the relevance of such studies is highly questionable[1].

The clonogenic assay is not without problems. The assay often involves plating of dilute concentrations of cells under conditions that are significantly different from those found in vivo. Furthermore, the in vitro conditions ignore the unique micro environmental parameters of a tumor that can be important contributors to treatment sensitivity such as oxygen and cell-to-cell contact. Several investigators have also pointed out that the long-term culture of cells in vitro can result in selection of resistant clones that are not reflective of the original cells in vivo. In some instances, it is thus desirable to use primary cells derived from tumors in vivo. In this case, it is important that these primary cells can also tolerate in vitro culture conditions without substantial death even without treatment. This can be assessed by the plating efficiency-a measure of the number of cells that retain clonogenic capacity in the absence of treatment. When this value is very low (less than $5 \%$ ), the predictive power of the clonogenic assay comes into question. However, such a low-plating efficiency is indicative of the fact that the cells do not tolerate in vitro culture well and thus place doubts on any in vitro assays that may be used with these cells. Thus, it is feel strongly that the clonogenic assay is far and away the best in vitro tool for assessing treatment response [1].

\section{In Vivo}

There is clearly a need to evaluate cellular treatment sensitivity within the context of the normal environment, a so-called in vivo assay. However, even in this context, several common assays can be highly biased or inappropriate for evaluating treatment response. In particular, assays that are based primarily on evaluating or comparing tumor size (or presence) at fixed times after treatment are difficult to interpret and heavily biased toward tumors that display rapid forms of cell death like apoptosis.

For example, investigators may treat either spontaneous or transplanted tumors and then evaluate the size of the tumor at one or more times after treatment. This type of experiment is heavily influenced by the intrinsic tumor growth rate, which is itself influenced by cell loss that occurs through death mechanisms such as apoptosis. A tumor that suffers a high loss factor will grow much more slowly than a similar tumor with a lower cell loss factor. To illustrate this problem, let consider two different tumors that behave identically with the exception that one has a much slower growth rate because of a higher rate of spontaneous apoptosis. If we treat each of these two tumors with a dose that produces the same amount of overall cell kill (the same level of survival), it will take substantially longer for the slow-growing tumor to reappear. This may cause misinterpretation of the treatment sensitivity as the "time to relapse" was significantly longer in the slowgrowing (apoptotically sensitive) tumor, even though treatment sensitivity and curability are identical. In fact, at any time after treatment, the slow-growing tumor will appear smaller than the other tumor leading one to this mistaken conclusion [1].

Misinterpretations of treatment sensitivity can also result from differences in the kinetics of cell death following treatment. Again, let consider a hypothetical situation in which two tumors have equal growth rates and equal overall responses to treatment. However, in this case, cell death in one of the tumors occurs principally through a rapid apoptotic mechanism and in the other through a much slower mechanism based on mitotic catastrophe. If one follows the size of the tumors after treatment the apoptotic tumor will regress rapidly because of the induction of apoptosis. The other tumor, although it will eventually show an equivalent loss in cell number, will not regress quickly and thus "appear" to respond more poorly. Again, assessment of tumor size at a fixed time after treatment will not yield the correct information on treatment sensitivity. Owing to both of the factors illustrated in these examples, comparison of tumor size at fixed times after treatment is nearly impossible to relate to overall treatment sensitivity [1].

A much better way to evaluate the treatment sensitivity of tumors is to measure the growth delay that results from treatment. The growth delay is a measure of the difference in times for treated and untreated tumors to reach a certain size (e.g., three times the starting volume). For this value to be meaningful, it must be evaluated at a sufficiently long time after treatment such that all forms of cell death have had an opportunity to have taken place. At this time, the tumor should be growing at a rate that is equivalent again to the untreated 
tumor. The difference in time required for the control and treated tumors to reach equivalent sizes directly reflects the percentage of cells that survived the treatment. This value is completely independent of the rate of tumor regression after treatment. To remove the problems associated with comparing tumors that have different intrinsic growth rates, one must use the specific growth delay. This is simply equal to the growth delay expressed as a fraction of the growth rate (e.g., time to reach three times starting volume) of the untreated tumor [1].

Although measurements of growth delay correct for the two errors outlined in our example, they do suffer from other potential problems. Tumor growth rate can be affected by treatment in ways that are unrelated to the overall amount of cell death of the tumor cells. For example, damage to tumor vasculature may slow tumor growth in a way that is unrelated to killing the tumor cells. This extra growth delay may be incorrectly interpreted as increased cell death even though no additional tumor cells may be killed.

The gold standard of all assays of treatment sensitivity is thus those that evaluates tumor cure. This is evaluated by a socalled TCD50 assay, in which the dose required to cure $50 \%$ of the tumors is calculated. In these experiments, cure typically follows a sigmoidal function of treatment dose. Although this is the best of all assays, there has been a steady decrease in its use. This is likely due to the fact that a large number of mice must be used to accurately define the dose-response relationship. Furthermore, this assay takes a comparatively long time, typically requiring many months to be sure that cure has been achieved. The TCD50 assay is somewhat analogous to the in vitro clonogenic assay, as it ultimately measures the probability that a single cell can survive and reform the tumor [1]. For example, at doses that will cure $50 \%$ of treated tumors, the average number of surviving cells per tumor is just slightly above1. The assay is unaffected by rates of tumor growth or rates of cell death. When performed correctly, this assay provides the ultimate measure of overall treatment sensitivity [1].

The TCD50 assay also allows one to consider the possible existence of tumor stem cells. This is an old concept that has received renewed interest and implies that only a small fraction of tumor cells are truly clonogenic and capable of unlimited proliferation. This concept may also explain the poor "plating efficiency" that has been observed when primary tumor cells are cultured in vitro. The bulk of the tumor cells thus represent somewhat more differentiated cells that lack this clonogenic capacity[1].

Obviously in such a case, it becomes important to evaluate the response of the tumor stem cells and not the response of the bulk of the tumor. As the TCD50 is influenced only by those cells that have the ability to both survive and reform the tumor, it accurately reflects the treatment sensitivity of the relevant cells in the tumor. The behavior of the non-stem cells in this case is irrelevant[1].

\section{Apoptotic Induction in Activated VECs[1]}

VEC apoptosis is essential during normal development and in the adult during phases of reproductive cycles and wound healing. As in other cell types, VEC apoptosis can occur through either the extrinsic (death receptor) or the intrinsic (mitochondrial) pathway. In addition to signals received by these pathways, four other essential signaling pathways that can regulate the fate of VECs, including paracrine, autocrine and endocrine factors (i.e. survival factor withdrawal), cell adhesion, blood flow and sheer stress, and the transcriptome and glycome. These pathways reflect the complex environment of the VECs $[1,7,9,32]$.

TSP-1 binds to the CD36 receptor on the VEC surface. PEDF also binds to the VEC surface; however, the surface receptor has not yet been identified. Following receptor binding, both TSP-1 and PEDF treatment stimulates an increase in Fas ligand (FasL/CD95 ligand) expression. As VEGF stimulates Fas expression, the activated VEC is primed to respond to the FasL. PEDF treatment has also been shown to decrease FLIP expression through PEDF-stimulated inhibition of NFATc2 $[1,8,36]$.

Decreased FLIP activity, as well as activation of Fas by the FasL, results in activated caspase- 8 and initiation of the apoptotic cascade which suggest that the levels of Fas/FasL and FLIP may represent two check points in VECs that determine survival or apoptosis $[1,7,8]$.

Among the other endogenous angiogenesis inhibitors, the mechanism of action of angiostatin has also been intensely studied. However, its biology is more complex as angiostatin, a kringle-containing fragment of plasminogen, exists as several isoforms.

Plasminogen has five kringle domains, and the angiostatin isoforms, generated by proteolysis, vary in their kringle domain content. Angiostatin isoforms can inhibit VEC migration, tube formation, and proliferation and can also induce apoptosis of VECs. And, for the angiostatin isoform containing kringles $1-3$, caspase activation is necessary for the anti-angiogenic activity. This suggests that both the intrinsic and extrinsic apoptotic pathways are activated by AS4.5 (an angiostatin isoform containing kringles 1-4 \& 85\% of kringle5) and may explain why AS4.5 is one of the most potent angiostatin isoforms and angiogenesis inhibitors identified to date $[1,7-9,15,23,32,33,39,40]$.

\section{Clinical Trials of Anti-Angiogenic Agents}

Many of the molecules regulating angiogenesis have now been identified, so it is necessary to begun the identification of unique properties of the tumor vasculature which distinguishes it from the normal vessels. As our basic understanding of tumor angio- genesis progresses, so does our ability to develop effective anti-angiogenic agents clinically, and a large number of anti-angiogenic agents are currently in clinical trials, as monotherapies and in combination with other anti-angiogenic or anti-cancer therapies. 
These agents vary in their molecular and cellular target(s) and are classified in different ways. The system used here classifies them as direct and indirect agents $[1,40]$.

\section{Direct Angiogenesis Inhibitors}

Direct angiogenesis inhibitors act to prevent the VECs from responding to pro- angiogenic stimuli or to directly induce VEC apoptosis. To prevent the VECs from responding to proangiogenic molecules, several different approaches have been taken, including sequestering the inducer in the tumor microenvironment, inhibition of binding of the inducer to its cognate receptor on the VECs, and inhibition of receptor signaling $[1,9,32]$.

Sequestration of inducers has been achieved using soluble receptors (decoy receptors) and inducer-binding antibodies. As VEGF is a potent angiogenic inducer and has been shown to be over expressed in many tumor types, it is the target for many agents of this type. Bevacuzimab (Avastin; Genentech, South San Francisco, CA, USA), a humanized anti-VEGF antibody, has recently gained FDA approval for treatment of some types of cancer. Bevacuzimab was first approved for use in colorectal cancer, and a recent phase III study combining Bevacuzimab with cytotoxic therapy showed improved survival in patients with metastatic colorectal cancer. Additional studies in nonsmall-cell lung cancer and metastatic breast cancer are in progress [1,29,38,41].

One theoretical disadvantage of many first generation agents of this class is that they target a single angiogenic inducer.Many tumors secrete multiple inducers, and cells within a given tumor can produce different angiogenic inducers; therefore, inhibiting one inducer may only select for the sub-population of tumor cells expressing different inducers or may not be effective against tumors that secrete multiple angiogenic inducers. Using these drugs in combination with other types of angiogenic inhibitors or with low dose (metronomic) chemotherapy, as do the Bevacuzimab studies should overcome this limitation. An advantage of this approach is that it would be effective against the activity of multiple inducers and thus multiple tumor types $[1,38,41]$.

\section{Indirect Angiogenesis Inhibitors}

Indirect angiogenesis inhibitors target tumor cells to block the expression and/or secretion of pro-angiogenic proteins that stimulate tumor-associated VECs $[1,7,8,32]$ or to increase expression of angiogenesis inhibitors. These agents appear to hold much promise as they can potentially act to change the expression pattern of multiple angio- genic mediators. One of the most thoroughly studied of this class of inhibitors is Iressa (Gefitinib/ZD1839; Astrazeneca, Wilmington, DE, USA). Iressa is a small molecule inhibitor that targets the tyrosine kinase activity of the epidermal growth factor receptor (EGFR) $[1,8]$, a member of the ErbB family of cell surface receptors [1].

Many indirect agents currently in development target several signaling pathways and thus are sometimes labeled multiple target agents. The advantage of this type of agent is that it may be effective against tumors with different types of mutations. In addition, some of these indirect agents can also act on the tumor-associated VECs if the targeted receptor is expressed in the VECs $[1,9,13,17,29,30,32,41,42]$.

\section{Conclusions and future possibilities}

Folkman first began championing his hypothesis that tumor growth was dependent on angiogenesis in 1971, and with the FDA approval of Avastin in 2004, anti-angiogenic therapies are finally a proven tool in the treatment of cancer. One of the challenges in trials of anti-angiogenic therapies has been in appropriate evaluation of efficacy at each stage of the clinical trials. With traditional therapies, phase I trials have focused on determining the MTD. However, with anti-angiogenic therapies, the MTD is not the optimal means of delivering these agents. Looking for tumor mass or tumor marker responses, while appropriate in phasell and III trials, is not a fair measure in phase I dose determining studies, and evaluating microvessel density or angiogenic mediator expression in tumor biopsies is not practical. Identification of such cancer biomarkers will not only aid in the clinical development of anti-angiogenic agents and other novel drug therapies but also may provide additional means by which to detect cancer before it becomes symptomatic.

In term of design of therapies in cancer treatment were based on the tissue of origin of the tumor, with all patients with the same tumor type receiving the same therapy. Later treatments considered the histology of the tumor, pathological grade, and stage classifications.

Around the turn of the century came a significant change to the traditional approaches. The successful development of Herceptin, an EGFR tyrosine kinase inhibitor, for therapy against ErbB2-positive breast cancer, gave birth to a new era in cancer drug design, tailoring therapies to the genetic profile of a given patient's tumor. Drugs that specifically target a signaling pathway known to be mutated in cancers are also called molecular targeting agents. Using genomic and/or proteomic screening, tumors could be profiled for genetic mutations and protein expression patterns, thus identifying the specific changes in angiogenic mediator expression. With this information, a tumor could be effectively treated with agents specifically targeting the identified culprits. Such designer therapies are the future of all cancer therapies and should prove to be much more effective than traditional approaches.

\section{Competing interests}

The authors declare that they have no competing interests.

\section{Article Hisotry}

Editor: Yuki Hamano, Chiba University Hospital, Japan.

EIC: G.J. Peters, VU University Medical Center, Netherlands.

Received 18 December 2011 Revised 21 January 2012

Accepted 31 January 2012 Published 14 February 2012

\section{Acknowledgement}

I want to give my sincere acknowledgement to Jennifer A Doll, Jennifer E Hobbs, Gerald A Soff, David A Gewirtz, Shawn E Holt and Steven Grant for preparing this article. 


\section{References}

1. Jennifer $A D$ et al. Role of Apoptosis in Anti Angiogenic Cancer Therapies. Senescence and Cancer .Humana Press Inc. pp 537-556.

2. Folkman, J. Tumor angiogenesis: therapeutic implications. $N$ Engl J Med 285, 1182-1186.

3. Kerbel R S, et al. 'Accidental' anti-angiogenic drugs. Anti-oncogene directed signal transduction inhibitors and conventional chemotherapeutic agents as examples. Eur J Cancer 36, 1248-57.

4. Browder, T. et al. Antiangiogenic scheduling of chemotherapy improves efficacy against experimental drug-resistant cancer. Cancer Res 60, 1878-1886.

5. Munoz-Chapuli, R. et al. Angiogenesis and signal transduction in endothelial cells. Cell Mol Life SCi 61, 2224-2243.

6. Nor, J. E. et al. Role of endothelial cell survival and death signals in angiogenesis. Angiogenesis 3, 101116.

7. Veitonmaki $\mathrm{N}$, et al. Endothelial cell surface ATP synthase-triggered caspase-apoptotic pathway is essential for k1-5-induced antiangiogenesis. Cancer Res 64, 3679-86

8. Chen, Y. H. et al. Angiostatin antagonizes the action of VEGF-A in human endothelial cells via two distinct pathways. Biochem Biophys Res Commun 310, 804-810.

9. Sharma $M R$, et al. Angiostatin-induced inhibition of endothelial cell proliferation/apoptosis is associated with the down-regulation of cell cycle regulatory protein cdk5. J Cell Biochem 91, 398409.

10. Pugh, C. W. et al. Regulation of angiogenesis by hypoxia: role of the HIF system. Nat Med 9, 677684.

11. Dameron, K. M. et al. Control of angiogenesis in fibroblasts by $\mathrm{p} 53$ regulation of thrombospondin-1. Science 265, 1582-1584.

12. Volpert, O. V. et al. Sequential development of an angiogenic phenotype by human fibroblasts progressing to tumorigenicity. Oncogene 14, 14951502.

13. Hobson, B. et al. Endothelial proliferation in tumours and normal tissues: continuous labelling studies. $\mathrm{Br} J$ Cancer 49, 405-413.

14. Denekamp J, Angiogenesis, neovascular proliferation and vascular patho- physiology as targets for cancer therapy. $\mathrm{Br} J$ Radiol 66, 181-96.

15. Carmeliet, P. Angiogenesis in health and disease. Nat Med 9, 653-660.

16. Bouck, N. P53 and angiogenesis. Biochim Biophys Acta 1287, 63-66.

17. Folkman, J. Angiogenesis and apoptosis. Semin Cancer Biol 13, 159-167.

18. Sridhar, S. S. et al. Targeting angiogenesis: a review of angiogenesis inhibitors in the treatment of lung cancer. Lung Cancer 42 Suppl 1, S81-91.

19. Folkman, J. Angiogenesis in cancer, vascular, rheumatoid and other disease. Nat Med 1, 27-31.

20. Boehm T, et al. Antiangiogenic therapy of experimental cancer does not induce acquired drug resistance. Nature 390, 404-7.

21. Kerbel, R. S. A cancer therapy resistant to resistance. Nature 390, 335-336.
22. Folkman, J. Endogenous angiogenesis inhibitors. APMIS 112, 496-507.

23. Gately, S. et al. Antiangiogenic scheduling of lower dose cancer chemotherapy. Cancer J 7, 427-436.

24. Kerbel, R. et al. Clinical translation of angiogenesis inhibitors. Nat Rev Cancer 2, 727-739.

25. Browder $\mathrm{T}$, et al. The hemostatic system as a regulator of angiogenesis. $\mathrm{J} \mathrm{Biol} \mathrm{Chem} \mathrm{275,} \mathrm{1521-}$ 4.

26. Stetler-Stevenson, W. G. et al. TIMP-2: an endogenous inhibitor of angiogenesis. Trends $\mathrm{Mol}$ Med 11, 97-103.

27. Lucas, R. et al. Multiple forms of angiostatin induce apoptosis in endothelial cells. Blood 92, 4730-4741.

28. Dhanabal, M. et al. Endostatin induces endothelial cell apoptosis. J Biol Chem 274, 11721-11726.

29. Hicklin, D. J. et al. Role of the vascular endothelial growth factor pathway in tumor growth and angiogenesis. J Clin Oncol 23, 1011-1027.

30. Cross, M. J. et al. VEGF-receptor signal transduction. Trends Biochem Sci 28, 488-494.

31. Dawson, D. W. et al. Pigment epithelium-derived factor: a potent inhibitor of angiogenesis. Science 285, 245-248.

32. Hanford, H. A. et al. Angiostatin(4.5)-mediated apoptosis of vascular endothelial cells. Cancer Res 63, 4275-4280.

33. Gately, S. et al. The mechanism of cancer-mediated conversion of plasminogen to the angiogenesis inhibitor angiostatin. Proc Natl Acad Sci U S A 94, 10868-10872.

34. Gately $S$, et al. Human prostate carcinoma cells express enzymatic activity that converts human plasminogen to the angiogenesis inhibitor, angiostatin. Cancer Res 56, 4887-90.

35. Davis, D. W. et al. Antiangiogenic tumor therapy. Biotechniques 34, 1048-1050, 1052, 1054 passim.

36. Hurwitz, $H$. Integrating the anti-VEGF-A humanized monoclonal antibody bevacizumab with chemotherapy in advanced colorectal cancer. Clin Colorectal Cancer 4 Suppl 2, S62-68.

37. Kerbel R S, et al. Possible mechanisms of acquired resistance to anti-angiogenic drugs: implications for the use of combination therapy approaches. Cancer Metastasis Rev 20, 79-86.

38. Penland, S. K. et al. Combining anti-VEGF approaches with oxaliplatin in advanced colorectal cancer. Clin Colorectal Cancer 4 Suppl 2, S74-80.

39. Gimbrone, M. A., Jr. et al. Tumor dormancy in vivo by prevention of neovascularization. J Exp Med 136, 261-276.

40. Folkman, J. Seminars in Medicine of the Beth Israel Hospital, Boston. Clinical applications of research on angiogenesis. $N$ Engl J Med 333, 1757-1763.

41. Rak, J. W. et al. Consequences of angiogenesis for tumor progression, metastasis and cancer therapy. Anticancer Drugs 6, 3-18.

42. Blood, C. H. et al. Tumor interactions with the vasculature: angiogenesis and tumor metastasis. Biochim Biophys Acta 1032, 89-118. 